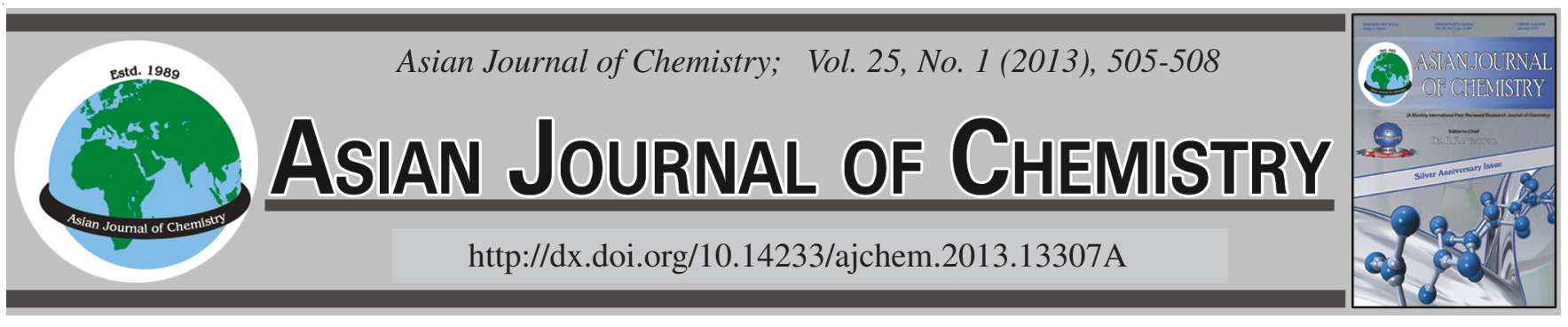

\title{
Synthesis and Decomposition of Parabanic Acid Derivatives
}

\author{
S.K. CHOI ${ }^{1}$, S.G. LEE ${ }^{1}$, D.I. JUNG ${ }^{1, *}$ and J.T. HAHN ${ }^{2}$
}

\author{
${ }^{1}$ Department of Chemistry, Dong-A University, Busan 604714, South Korea \\ ${ }^{2}$ Department of Beautycare, Youngdong University, Youngdong 370701, South Korea \\ *Corresponding author: Fax: +82 51 2007249; Tel: +82 51 2007249; E-mail: dijung@dau.ac.kr
}

Key Words: Parabanic acid, Benzimidazole, 1,2-Benzisothiazol-3-one-1,1-dioxide, Agrochemical fungicide, Urea derivatives.

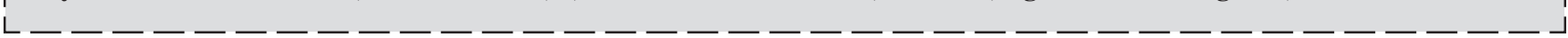

\section{INTRODUCTION}

Parabanic acid(imidazolidine-2,4,5-trione, oxalylurea) resulting from the oxidation of biological fundamental compounds such as uric acid, guanine, uracil and alloxanic acid has attracted the attention of agricultural and medicinal chemists for many decades ${ }^{1,2}$. Parabanic acid derivatives are useful phamaceuticals for the treatment of diabetic complications such as diabetic neuropathy, diabetic cataracts and diabetic dermotopathy ${ }^{3-6}$. Further, parabanic acid derivatives are known for their herbicidal, plant growth regulating and fungicidal properties $^{7}$. Polymers containing imidazolidine-2,4,5-trione (parabanic) rings are known as highly thermally stable polymers with improved chemical resistance in organic solvents ${ }^{8-13}$. Recently, many marine imidazol alkaloids have been isolated from sponges and their antitumor and antibacterial activities have also been identified ${ }^{14,15}$. In our previous works to obtain new agrochemicals, we reported the synthesis of new saccharin derivatives containing the 2,4,5-imidazolidinetrione group ${ }^{16}$. As a part of research program related to the synthetic study of pharmacologically and agrochemically important imidazolidines $^{16}$, we chose to identify imidazolidine-2,4,5-trione and imidazolidine-2,4,5-trione,2-thioxo-imidazolidine-4,5-dione, benzimidazole, orsaccharin rings as active components for the desired property, the synthesis of (imidazolidine-2,4,5-trionyl) imidazolidine-2,4,5-triones (1), 2,4,5-imidazolidinetrionyl1,2-benzisothiazol-3-one-1,1-di-oxide 2, 4,5-dioxo-2-thioxoimidazolidin-1-ylmethyl-imidazolidine-2,4,5-triones (2) and 1-benzoimidazole-1-yl-3-imidazolidine-2,4,5-trione (3) are already reported.

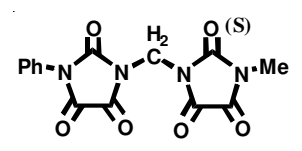

1

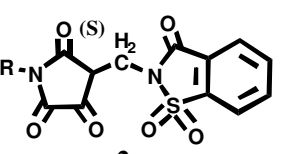

2

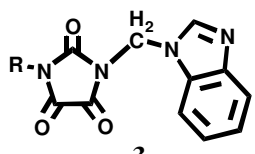

3

\section{EXPERIMENTAL}

Melting points were determined on a Büchi 510 capillary melting point apparatus and uncorrected. Infrared spectra were recorded on a Perkin-Elmer 683 spectro-photometer. NMR spectra were recorded on a Varian XL-300 or Brüker AC 200 FT-NMR spectrometer in $\mathrm{CDCl}_{3}$ containing $\mathrm{Me}_{4} \mathrm{Si}$ as an internal reference. Mass spectra were obtained by using JEOL JMS DX 303 or HP 5892 Mass Spectrometer.

General procedure for the synthesis of 6,7: To a solution of 1-chloromethyl-imidazolidine-2,4,5-triones (4) (or 1-chloromethyl-2-thioxo-imidazolidine-4,5-diones (5), $1.13 \times 10^{-2} \mathrm{~mol}$ ) in dry THF $(15 \mathrm{~mL})$ under nitrogen at room temperature was added solution of 2,4,5-imidazolidinetriones (or 2-thioxoimidazolidine-4,5-diones, $1.13 \times 10^{-2} \mathrm{~mol}$ ) and triethylamine $(0.4 \mathrm{~mL})$ in dry THF $(15 \mathrm{~mL})$. The reaction mixture was stirred at room temperature for $0.5 \mathrm{~h}$. After $0.5 \mathrm{~h}$, the reaction mixture was refluxed at $60-65^{\circ} \mathrm{C}$ for $7 \mathrm{~h}$. The reaction mixture was cooled again to room temperature and THF $(75 \mathrm{~mL})$ was added. The combined organic layers were dried over anhydrous $\mathrm{Na}_{2} \mathrm{SO}_{4}$, filtered and concentrated in vacuo. The residue was purified by flash column chromatography on silica gel eluted with only $\mathrm{CH}_{2} \mathrm{Cl}_{2}$, to provide products $\mathbf{6 , 7}$.

1-[(3-Phenylimidazolidine-2,4,5-trionyl)methyl]-3methyl-imidazolidine-2,4,5-trione (6): m.p. $247-249^{\circ} \mathrm{C}$; 
${ }^{1} \mathrm{H}$ NMR (200 MHz, DMSO) 3.08 (s, 3H), 5.43 (s, 2H), 7.52 $(\mathrm{m}, 5 \mathrm{H}) ;{ }^{13} \mathrm{C}$ NMR (50 MHz, DMSO) 157.2, 156.1, 155.9, 155.7, 152.8, 151.7, 130.2, 129.3, 128.9, 126.5, 40.6, 24.5; GC/MS m/z $330\left(\left[\mathrm{M}^{+}\right]\right)$; Anal. calcd. for $\mathrm{C}_{14} \mathrm{H}_{10} \mathrm{~N}_{4} \mathrm{O}_{6}:$ C, 50.92; H, 3.05; N, 16.96. Found : C, 50.95; H, 3.04; N, 16.94.

1-[(4,5-Dioxo-3-phenyl-2-thioxoimidazolidin-1yl)methyl]-3-ethyl-imidazolidine-2,4,5-trione (7): m.p. 217$218^{\circ} \mathrm{C}$; ${ }^{1} \mathrm{H}$ NMR (200 MHz, DMSO) 1.11 (t, $\left.J=7.1 \mathrm{~Hz}, 6 \mathrm{H}\right)$, $3.51(\mathrm{~d}, J=6.9 \mathrm{~Hz}, 2 \mathrm{H}), 5.53(\mathrm{~s}, 2 \mathrm{H}), 3.80(\mathrm{~d}, J=7.0 \mathrm{~Hz}, 2 \mathrm{H})$; ${ }^{13} \mathrm{C}$ NMR (50 MHz, DMSO) 180.1, 156.7, 156.0, 154.9, 154.7, 152.4, 43.6, 36.6, 33.8, 12.9, 12.5; GC/MS m/z 360([M+ $])$; Anal. calcd. for $\mathrm{C}_{15} \mathrm{H}_{12} \mathrm{~N}_{4} \mathrm{O}_{5} \mathrm{~S}: \mathrm{C}, 50.00 ; \mathrm{H}, 3.36 ; \mathrm{N}, 15.55$. Found : C, 50.03; H, 3.35; N, 15.59.

The typical experimental procedure for 1-phenyl-3-(1,1,3trioxox-1,3-dihydro-16-benzo[d]isothiazol-2-ylmethyl)imidazolidine-2,4,5-trione (6) is as follows: To a solution of 1-chloromethyl-3-phenylimidazolidine-2,4,5-trione (4c) (2.38 $\mathrm{g}, 10 \mathrm{mmol})$ in dry THF $(15 \mathrm{~mL})$ under nitrogen at room temperature was added solution of saccharin $(2.01 \mathrm{~g}, 11 \mathrm{mmol})$ and triethylamine $(1.2 \mathrm{~mL})$ in dry THF $(15 \mathrm{~mL})$. The reaction mixture was stirred at room temperature for $0.5 \mathrm{~h}$. After $0.5 \mathrm{~h}$, the reaction mixture was refluxed at $55-60{ }^{\circ} \mathrm{C}$ for $5 \mathrm{~h}$. The reaction mixture was cooled again to room temperature and THF $(50 \mathrm{~mL})$ was added. The combined organic layers were dried over anhydrous $\mathrm{Na}_{2} \mathrm{SO}_{4}$, filtered and concentrated in vacuo. The residue was purified by flash column chromatography on silica gel eluted with only $\mathrm{CH}_{2} \mathrm{Cl}_{2}$, to provide the 1-phenyl-3-(1,1,3-trioxo-1,3-dihydro-16-benzo[d]isothiazol2-yl-methyl)-imidazolidine-2,4,5-trione $\mathbf{6}$ as a white crystalline solid (1.93 g, $50 \%)$. m.p. 191-192 ${ }^{\circ} \mathrm{C}$; ${ }^{1} \mathrm{H}$ NMR $(200$ $\left.\mathrm{MHz}, \mathrm{CDCl}_{3}\right) 5.84$ (s, 2H, $\mathrm{CH}_{2}$ ), 7,4-8.1 (m, 9H, phenyl); Mass $\mathrm{m} / \mathrm{z}$ (rel. intensity, \%) 385 ([M+], 40), 196 (100), 91, 77.

The typical experimental procedure for 1-ethyl-2-thioxo3-(1,1,3-tri-oxo-1,3-dihydro-16-benzo[d]isothiazol-2ylmethyl)-imidazolidine-4,5-dione (7) is as follows: To a solution of 1-ethyl-2-thioxo-imidazolidine-4,5-dione (5b) (1 g, $4.8 \mathrm{mmol})$ in the dry THF $(10 \mathrm{~mL})$ under nitrogen at room temperature was added solution of saccharin $(1.5 \mathrm{~g}, 8.2 \mathrm{mmol})$ and triethylamine $(1.1 \mathrm{~mL})$ in the dry THF $(10 \mathrm{~mL})$. The reaction mixture was stirred at room temperature for $0.5 \mathrm{~h}$. After $0.5 \mathrm{~h}$, the mixture was refluxed $55-60{ }^{\circ} \mathrm{C}$ for $5 \mathrm{~h}$. The reaction mixture was cooled again to room temperature and THF $(50 \mathrm{~mL})$ was added. The combined organic layers were dried over anhydrous $\mathrm{Na}_{2} \mathrm{SO}_{4}$, filtered and concentrated in vасио. The residue was purified by flash column chromatography on silica gel eluted with only $\mathrm{CH}_{2} \mathrm{Cl}_{2}$, to provide the 1ethyl-2-thioxo-3-(1,1,3-trioxo-1,3-dihydro-16-benzo[d] isothiazol-2-ylmethyl)-imidazolidine-4,5-dione (7) as a yellow crystalline solid (1.68 g, $49 \%$ ). m.p. $157-158{ }^{\circ} \mathrm{C} ;{ }^{1} \mathrm{H}$ NMR (200 MHz, acetone- $\left.d_{6}\right) 1.17$ (t, 3H, - $\mathrm{CH}_{3}$ ), 3.99-4.06 (q, 2H, $-\mathrm{CH}_{2}$ ), 6.04 (s, 2H, N-CH ${ }_{2}-\mathrm{N}$ ), 8.05-8.20 (m, 4H, phenyl); IR $\left(\mathrm{KBr}, v_{\max }, \mathrm{cm}^{-1}\right): 1780,1740,1720,1390,1330,1280,1240$, 1175; Mass m/z (rel. intensity, \%) $352\left(\left[\mathrm{M}^{+}\right]\right)$.

1-Chloromethyl-3-methyl-imidazolidine-2,4,5-trione (4a): Yield $50 \%$; m.p. $149-150{ }^{\circ} \mathrm{C}$; IR $\left(\mathrm{KBr}, \mathrm{v}_{\max }, \mathrm{cm}^{-1}\right)$ : 1731 , 1459, 1407, 1306, 1129; Mass $\mathrm{m} / \mathrm{z}$ (rel. intensity, \%) 179 $\left([\mathrm{M}+2]^{+}, 5\right), 176\left([\mathrm{M}]^{+}, 20\right), 141$ (100), 113 (30), 94 (5), 70 (35), 56 (85); $\left.{ }^{1} \mathrm{H} \mathrm{NMR} \mathrm{(200} \mathrm{MHz,} \mathrm{CDCl}_{3}\right) 3.23$ (s, 3H, $\mathrm{CH}_{3}$ ), $5.40\left(\mathrm{~s}, 2 \mathrm{H}, \mathrm{CH}_{2} \mathrm{Cl}\right)$.
1-Chloromethyl-3-ethyl-imidazolidine-2,4,5-trione (4b): Yield $64 \%$; m.p. 83-84 ${ }^{\circ} \mathrm{C}$; IR (KBr, $\left.v_{\max }, \mathrm{cm}^{-1}\right) 2982$, 2865, 1735, 1409, 1298, 1208, 1128; Mass $m / z$ (rel. intensity, \%) $192\left([\mathrm{M}+1]^{+}, 5\right), 190\left([\mathrm{M}-1]^{+}, 10\right), 175(2), 162(1), 154$ (30), 127 (15), 99 (10), 70 (45), 56 (100); ${ }^{1} \mathrm{H}$ NMR (200 MHz, $\left.\mathrm{CDCl}_{3}\right)$ 1.27-1.34 (t, 3H, $\mathrm{CH}_{3}$ ), 3.70-3.81 (q, $2 \mathrm{H}, \mathrm{CH}_{2} \mathrm{Cl}$ ), 5.39 (s, $2 \mathrm{H}, \mathrm{CH}_{2} \mathrm{Cl}$ ).

1-Chloromethyl-3-phenyl-imidazolidine-2,4,5-trione (4c): Yield $70 \%$; m.p. $130-131^{\circ} \mathrm{C}$; IR (KBr, $\left.v_{\max }, \mathrm{cm}^{-1}\right) 1780$, 1730, 1500, 1440, 1295, 1190; Mass $m / z$ (rel. intensity, \%) $238\left([\mathrm{M}]^{+}, 27\right), 119$ (100), 91 (23); ${ }^{1} \mathrm{H}$ NMR (200 MHz, $\left.\mathrm{CDCl}_{3}\right)$ $5.6\left(\mathrm{~s}, 2 \mathrm{H}, \mathrm{CH}_{2} \mathrm{Cl}\right), 7.4-7.5$ ( $\mathrm{m}, 5 \mathrm{H}$, phenyl).

1-Methyl-3-(1,1,3-trioxo-1,3-dihydro-16-benzo[d]isothiazol-2-yl-methyl)-imidazolidine-2,4,5-trione (8a): Yield $40 \%$; m.p. $192-193{ }^{\circ} \mathrm{C}$; IR (KBr, $\left.v_{\max }, \mathrm{cm}^{-1}\right): 1749,1453,1340$, 1291, 1245, 1179; Mass $m / z$ (rel. intensity, \%) $323\left([\mathrm{M}]^{+}, 1\right)$, 259 (15), 223 (4), 196 (100), 174 (20), 169 (17), 132 (20), 121 (5), 104 (22), 76 (15), 70 (9); ${ }^{1} \mathrm{H}$ NMR (200 MHz, Acetone- $d_{6}$ ) $3.14\left(\mathrm{~s}, 3 \mathrm{H}, \mathrm{CH}_{3}\right), 5.70$ (s, 2H, $\left.\mathrm{CH}_{2}\right), 8.05-8.21$ (m, 4H, phenyl).

1-Ethyl-3-(1,1,3-trioxo-1,3-dihydro-16-benzo-[d] isothiazol-2-yl-methyl)-imidazolidine-2,4,5-trione (8b): Yield $54 \%$; m.p. $182-183^{\circ} \mathrm{C}$; IR $\left(\mathrm{KBr}, \mathrm{v}_{\max }, \mathrm{cm}^{-1}\right)$ : 2981, 2885 , 1746, 1425, 1340, 1293, 1251, 1180, 1115; Mass $\mathrm{m} / \mathrm{z}$ (rel. intensity, \%) 337 ([M] $\left.]^{+}, 2\right), 273$ (8), 223 (4), 196 (100), 174 (15), 169 (14), 132 (20); ${ }^{1} \mathrm{H}$ NMR (200 MHz, Acetone- $d_{6}$ ) 1.18-1.28 (t, 3H, $\left.\mathrm{CH}_{3}\right), 3.64-3.75$ (q, $2 \mathrm{H}, \mathrm{CH}_{2}$ ), 5.70 (s, $2 \mathrm{H}$, $\left.\mathrm{N}-\mathrm{CH}_{2}-\mathrm{N}\right), 8.02-8.22$ (m, 4H, phenyl).

1-Phenyl-3-(1,1,3-trioxo-1,3-dihydro-16-benzo[d]isothiazol-2-yl-methyl)-imidazolidine-2,4,5-trione (8c): Yield $50 \%$; m.p. 191-192 ${ }^{\circ} \mathrm{C}$; IR (KBr, $\left.v_{\max }, \mathrm{cm}^{-1}\right): 1785,1750,1410$, 1330, 1290, 1250; Mass $m / z$ (rel. intensity, \%) 385 ([M] $\left.]^{+}, 40\right)$, 196 (100), 169 (20), 119 (80), 91 (45), 77 (33); ${ }^{1} \mathrm{H}$ NMR (200 $\left.\mathrm{MHz}, \mathrm{CDCl}_{3}\right) 5.84$ (s, 2H, $\mathrm{CH}_{2}$ ), 7.4-8.1 (m, 9H, phenyl).

1-Chloromethyl-3-methyl-2-thioxo-imidazolidine-4,5dione (5a): Yield $45 \%$; m.p. 97-98 ${ }^{\circ} \mathrm{C}$; IR $\left(\mathrm{KBr}, v_{\max }, \mathrm{cm}^{-1}\right)$ : 1784, 1772, 1405, 1378, 1354; Mass $m / z$ (rel. intensity, \%) $192\left([\mathrm{M}]^{+}\right), 1 ;{ }^{1} \mathrm{H}$ NMR (200 MHz, Acetone- $\left.d_{6}\right) 3.38(\mathrm{~s}, 3 \mathrm{H}$, $\left.\mathrm{CH}_{3}\right), 5.76\left(\mathrm{~s}, 2 \mathrm{H}, \mathrm{CH}_{2}\right)$.

1-Chloromethyl-3-ethyl-2-thioxo-imidazolidine-4,5dione (5b): Yield $45 \%$; m.p. $108-109^{\circ} \mathrm{C}$; IR $\left(\mathrm{KBr}, \mathrm{v}_{\max }, \mathrm{cm}^{-1}\right)$ : 1780, 1740, 1402, 1370, 1230, 1135; Mass $m / z$ (rel. intensity, \%) $206\left([\mathrm{M}]^{+}\right) ;{ }^{1} \mathrm{H}$ NMR $\left(200 \mathrm{MHz}\right.$, Acetone- $\left.d_{6}\right)$ 1.21-1.28 (t, $3 \mathrm{H}, \mathrm{CH}_{3}$ ), 3.95-4.06 (q, 2H, $\left.\mathrm{CH}_{2}\right), 5.75$ (s, 2H, $\mathrm{CH}_{2} \mathrm{Cl}$ ).

1-Chloromethyl-3-phenyl-2-thioxo-imidazolidine-4,5dione (5c): Yield $40 \%$; m.p. $149-150^{\circ} \mathrm{C}$; IR $\left(\mathrm{KBr}, \mathrm{v}_{\max }, \mathrm{cm}^{-1}\right)$ : 1780, 1650, 1520, 1500, 1400, 1230; Mass $m / z$ (rel. intensity, \%) $254\left([\mathrm{M}]^{+}\right) ;{ }^{1} \mathrm{H}$ NMR (200 MHz, Acetone- $\left.d_{6}\right) 5.87$ (s, 2H, $\mathrm{CH}_{2} \mathrm{Cl}$ ), 7.43-7.60 (m, 5H, phenyl).

1-Methyl-2-thioxo-3-(1,1,3-trioxo-1,3-dihydro-16benzo[d]isothiazol-2-ylmethyl)-imidazolidine-4,5-dione (9a): Yield $40 \%$; m.p. $173-174^{\circ} \mathrm{C}$; IR (KBr, $\left.v_{\max }, \mathrm{cm}^{-1}\right)$ : 1780 , 1400, 1340, 1290, 1250, 1170, 1100; Mass $\mathrm{m} / z$ (rel. intensity, \%) $339\left([\mathrm{M}]^{+}\right) ;{ }^{1} \mathrm{H}$ NMR $\left(200 \mathrm{MHz}\right.$, Acetone- $\left.d_{6}\right) 3.39(\mathrm{~s}, 3 \mathrm{H}$, $\mathrm{CH}_{3}$ ), 6.04 (s, 2H, N-CH$-\mathrm{N}$ ), 8.10-8.17 (m, 4H, phenyl).

1-Ethyl-2-thioxo-3-(1,1,3-trioxo-1,3-dihydro-16benzo[d]isothiazol-2-yl-methyl)-imidazolidine-4,5-dione (9b): Yield $49 \%$; m.p. $157-158^{\circ} \mathrm{C}$; IR (KBr, $\left.v_{\max }, \mathrm{cm}^{-1}\right)$ : 1780 , 1740, 1720, 1390, 1330, 1280, 1240, 1175; Mass $\mathrm{m} / \mathrm{z}$ (rel. intensity, \%) $353\left([\mathrm{M}]^{+}\right)$; ${ }^{1} \mathrm{H}$ NMR $\left(200 \mathrm{MHz}\right.$, Acetone- $\left.d_{6}\right)$ 
1.17 (s, 3H, $\left.\mathrm{CH}_{3}\right), 3.99-4.06\left(\mathrm{~s}, 2 \mathrm{H}, \mathrm{CH}_{2}\right), 6.04$ (s, 2H, N$\left.\mathrm{CH}_{2}-\mathrm{N}\right), 8.05-8.20$ (m, $4 \mathrm{H}$, phenyl).

1-Phenyl-2-thioxo-3-(1,1,3-trioxo-1,3-dihydro-16benzo[d]isothiazol-2-ylmethyl)-imidazolidine-4,5-dione (9c): Yield $30 \%$; m.p. 170-171 ${ }^{\circ} \mathrm{C}$; IR $\left(\mathrm{KBr}, \mathrm{v}_{\max }, \mathrm{cm}^{-1}\right)$ : 1785, 1720, 1400, 1370, 1360, 1300, 1250, 1180; Mass $\mathrm{m} / \mathrm{z}$ (rel. intensity, \%) $402\left([\mathrm{M}]^{+}\right) ;{ }^{1} \mathrm{H} \mathrm{NMR}\left(200 \mathrm{MHz}\right.$, acetone- $\left.d_{6}\right) 6.58$ (s, 2H, $\mathrm{CH}_{2}$ ), 7.84-8.63 (m, 9H, phenyl).

1-Benzoimidazole-1-yl-methyl-3-imidazolidine-2,4,5trione (10a): $\mathrm{R}_{\mathrm{f}} 0.7$ (TLC eluent; $n$-Hexane : EtOAc $=1: 25$, v/v); yield $75 \%$; m.p. $188-189^{\circ} \mathrm{C}$; IR $\left(\mathrm{KBr}, \mathrm{v}_{\max }, \mathrm{cm}^{-1}\right)$ : 3846 , 1778, 1452, 1344, 1244, 756; Mass $m / z$ (rel. intensity, \%) 258 (100), 173 (7), 145 (9), 131 (100), 118 (15), 104 (12), 90 (15), 77 (20), 56 (64), 51 (12); ${ }^{1} \mathrm{H}$ NMR (200 MHz, $\left.\mathrm{CDCl}_{3}\right) 3.18$ (s, $\left.3 \mathrm{H}, \mathrm{CH}_{3}\right), 6.18$ (s, 2H, N-CH$\left.-\mathrm{N}\right), 7.25-7.78$ (m, 4H, phenyl), 8.2 (s, $1 \mathrm{H}$, benzimidazole).

1-Benzoimidazole-1-yl-ethyl-3-imidazolidine-2,4,5trione (10b): $\mathrm{R}_{\mathrm{f}} 0.7$ (TLC eluent; $n$-Hexane: EtOAc $=1: 25$, v/v); yield $50 \%$; m.p. $179-180^{\circ} \mathrm{C}$; IR $\left(\mathrm{KBr}, \mathrm{v}_{\max }, \mathrm{cm}^{-1}\right)$ : 3541 , 2984, 1736, 1425, 1242, 796; Mass $m / z$ (rel. intensity, \%) 272 (93), 245 (2), 173 (5), 145 (7), 131 (100), 118 (11), 104 (8), 90 (10), 77 (14), 56 (57), 51 (8); ${ }^{1} \mathrm{H}$ NMR (200 MHz, $\left.\mathrm{CDCl}_{3}\right)$ 1.25 (t, 3H, CH3), 3.65 (q, 2H, $\mathrm{CH}_{2}$ ), 5.97 (s, 2H, N-CH $-\mathrm{N}$ ), 7.25-7.82 (m, 4H, phenyl), 8.19 (s, 1H, benzimidazole).

1-Benzoimidazole-1-yl-phenyl-3-imidazolidine-2,4,5trione (10c): $\mathrm{R}_{\mathrm{f}} 0.7$ (TLC eluent; $n$-Hexane : EtOAc $=1: 25$, v/v); yield $45 \%$; m.p. $187-188^{\circ} \mathrm{C}$; IR (KBr, $\left.v_{\max }, \mathrm{cm}^{-1}\right) 3884$, 2924, 1767, 1741, 1242; Mass $m / z$ (rel. intensity, \%) 320 (61), 173 (5), 145 (4), 131 (100), 119 (7), 91 (8), 77 (12), 64 (6), 56 (26); ${ }^{1} \mathrm{H}$ NMR (200 MHz, $\left.\mathrm{CDCl}_{3}\right) 6.15$ (s, 2H, N-CH $\left.-\mathrm{N}\right), 7.15-$ 7.85 ( $\mathrm{m}, 9 \mathrm{H}$, phenyl), 8.25 (s, 1H, benzimidazole).

1-Phenylurea (11): Isolated yield $41 \% ; \mathrm{R}_{\mathrm{f}} 0.35$ (TLC eluent; EtOAc : $n$-Hexane $=1: 5, \mathrm{v} / \mathrm{v}) ;$ Mass $m / z($ rel. intensity, $\%)$ 135; ${ }^{1} \mathrm{H}$ NMR $\left(200 \mathrm{MHz}, \mathrm{CDCl}_{3}\right) 6.20$ (s, 1H), 7.19 (m, 1H), 7.43 (m, 2H), 7.61 (dd, 2H), 8.9 (s, 1H); ${ }^{13} \mathrm{C} \mathrm{NMR} \mathrm{(50} \mathrm{MHz,}$ $\left.\mathrm{CDCl}_{3}\right)$ 121.6, 128.0, 128.9, 139.4; Anal. calcd. for $\mathrm{C}_{7} \mathrm{H}_{8} \mathrm{~N}_{2} \mathrm{O}$ : C, 61.75; H, 5.92; N, 3.47; Found : C, 61.73; H, 5.91; N, 3.44.

1-Ethyl-3-[(3-ethylthioureido)methyl]urea (12): Isolated yield $26 \% ; \mathrm{R}_{\mathrm{f}} 0.35$ (TLC eluent; EtOAc : $n$-Hexane $=1$ : 8, v/v); Mass m/z (rel. intensity, \%) 204 (29), 167 (29), 149 (100), 104 (43), 76 (38), 70 (55), 55 (100); ${ }^{1} \mathrm{H}$ NMR (200 $\left.\mathrm{MHz}, \mathrm{CDCl}_{3}\right) 6.20$ (s, 1H), 7.19 (m, 1H), 7.43 (m, 2H), 7.61 (dd, 2H), 8.9 (s, 1H); ${ }^{13} \mathrm{C}$ NMR (50 MHz, $\left.\mathrm{CDCl}_{3}\right)$ 14.5, 15.3, 34.2, 40.2, 62.3, 157.9, 182.2; Anal. calcd. for $\mathrm{C}_{7} \mathrm{H}_{16} \mathrm{~N}_{4} \mathrm{O}-\mathrm{S}$ : C, 41.15; H, 7.89; N, 27.40; O, 7.83; S, 15.7; Found: C, 41.13; H, 7.88; N, 27.40; O, 7.79; S, 15.64.

1-Ethyl-3-[(3-methylthioureido)methyl]urea (13): Isolated yield $23 \% ; \mathrm{R}_{\mathrm{f}} 0.35$ (TLC eluent; EtOAc: $n$-Hexane $=1: 8, \mathrm{v} / \mathrm{v}$ ); Mass $m / z$ (rel. intensity, \%) 190 (5), 119 (100), 91 (95), 77 (5), 64 (81), 51 (31); ${ }^{1} \mathrm{H}$ NMR (200 MHz, $\left.\mathrm{CDCl}_{3}\right) 6.20$ (s, 1H), $7.19(\mathrm{~m}, 1 \mathrm{H}), 7.43(\mathrm{~m}, 2 \mathrm{H}), 7.61(\mathrm{dd}, 2 \mathrm{H}), 8.9(\mathrm{~s}, 1 \mathrm{H}) ;{ }^{13} \mathrm{C} \mathrm{NMR}$ (50 $\left.\mathrm{MHz} \mathrm{CDCl}_{3}\right)$ 14.5, 31.0, 34.3, 62.3, 157.7, 182.2; Anal. calcd. for $\mathrm{C}_{6} \mathrm{H}_{14} \mathrm{~N}_{4} \mathrm{OS}$ : C, 37.88; H, 7.42; N, 29.45; O, 8.41; S, 16.85; Found: C, 37.84; H, 7.40; N, 29.41; O, 8.40; S, 16.82.

\section{RESULTS AND DISCUSSION}

The basic catalyzed condeusation between imidazolidin2,4,5-triones and paraformaldehyde in aqueous solution yielded a mixture of the expected $N$-hydroxy methyl dericatives and imidazolidine-2,4,5-triones. However, the instability of $N$-hydroxy methyl derivatives made it isolation very difficult. The use of column chromatography as a method of purification was not successful, whatever the eluent or support(silica gel, alumina) was used, because the $\mathrm{R}_{\mathrm{f}}$ value was the same for the two compounds. For this reaction, the next chloriration step, using a large excess of thionyl chloride, was realized starting directly from a mixture of $N$-hydroxy methyl derivatives and imidazolidine-2,4,5-triones. The chlorinated precursors $\mathbf{4 , 5}$ were easily isolated by column chromatography and the product had a much higher $\mathrm{R}_{\mathrm{f}}$ value the starting material.
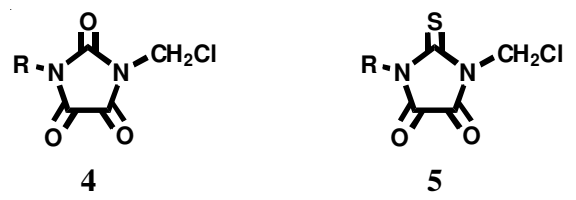

When the chlorinated products $\mathbf{4}$ and $\mathbf{5}$ were allowed to react with $N$-methylimidazolidine-2,4,5-trione and $N$-phenylimidazolidine-2,4,5-trione, respectively, 1-[(3-phenylimidazolidine-2,4,5-trionyl)methyl]-3-methyl-imidazolidine-2,4,5trione 6 and 1-[(4,5-dioxo-3-phenyl-2-thioxoimidazolidin-1yl)methyl]-3-ethylimidazolidine-2,4,5-trione 7 were obtained in good yields, as shown in Table- 1 . When chlorinated products $\mathbf{4}$ and $\mathbf{5}$ were allowed to react with 1,2-benzothiazole-3-one1,1-dioxide by same methods, saccharin derivatives $\mathbf{8 , 9}$ containing imidazolidine-2,4,5-trionyl, 2-thioxo-imidaz-olidine4,5-dionyl groups were obtained in good yields as shown in the Table-1. We also tried to obtain various parabanic acid derivatives from the reactions of the chlorinated reactants $\mathbf{4 , 5}$ with benzimidazole. 1-Benzoimidazole-1-yl-methyl-3imidazolidine-2,4,5-trione 10a (yield, $75 \%$ ), 1-benzoimi-

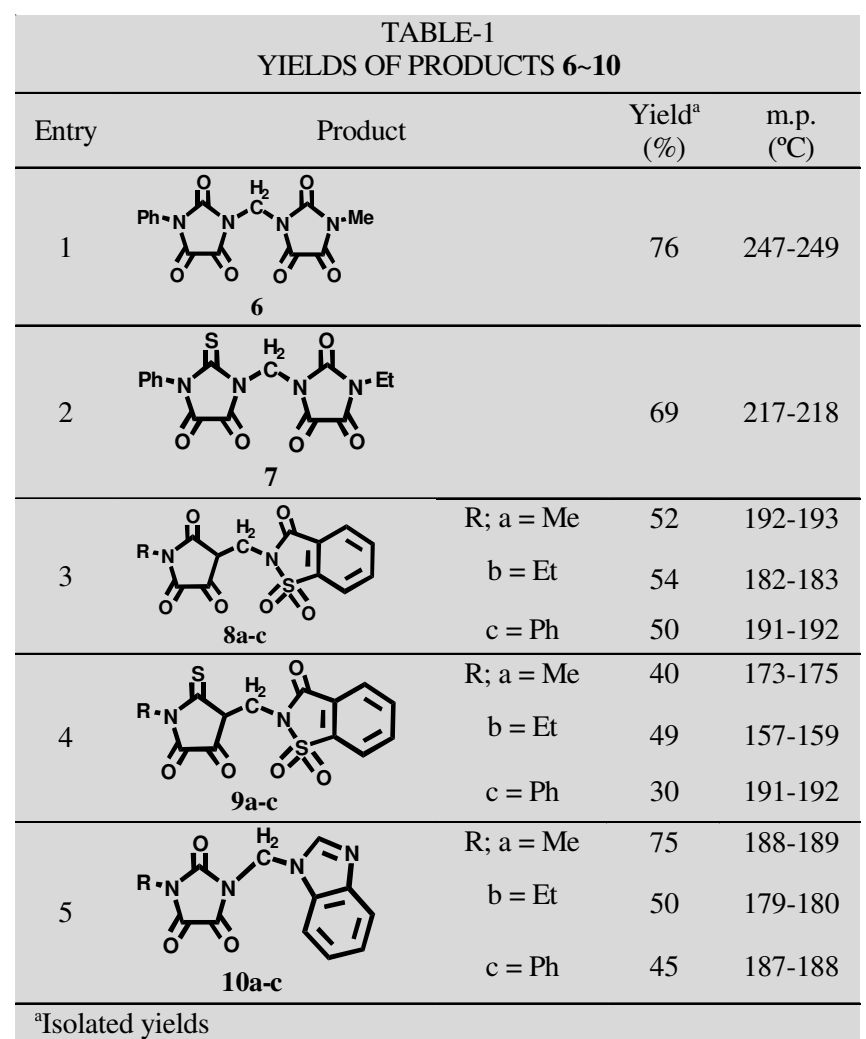


dazole-1-yl-ethyl-3-imidazolidine-2,4,5-trione (10b) (yield, $50 \%$ ) and 1-benzoimidazole-1-yl-phenyl-3-imidazolidine2,4,5-trione (10c) (yield, $45 \%$ ) were obtaines in good yields, as shown in Table-1.

Biological tests for tesring the phytocidal, herbicidal and insecticidal properties of the new synthesized compounds (610) are currently in progress. As a part of a research program related to the synthesis study of bioactive organo chemicals, we also report the synthesis of Di-urea derivatives in the reaction of 1-phenylimidazolidine-2,4,5-trione, 1-ethyl-3-[(3-ethyl-4,5dioxo-2-thioxoimidazolidin-1-yl)methyl]-imidazolidine-2,4,5trione and 1-ethyl-3-[(3-methyl-4,5-dioxo-2-thioxoimidazolidin-1-yl)methyl]-imidazolidine-2,4,5-trione with hydrolysis in basic solution through ultrasound.

1-Phenylurea (11), 1-ethyl-3-[(3-ethylthioureido)methyl]urea (12) and 1-ethyl-3-[(3-methylthioureido)methyl]urea (13) were obtained in good yields as shown in Table-2.

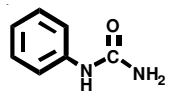

11

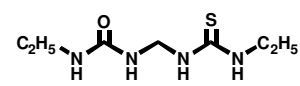

12

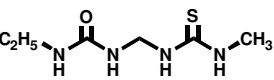

13

TABLE-2

YIELDS OF OBTAINED PRODUCTS 11,

12, 13 THROUGH ULTRASOUND

\begin{tabular}{|c|c|c|c|}
\hline Entry & Product & $\begin{array}{l}\text { Reaction } \\
\text { time (h) }\end{array}$ & $\begin{array}{c}\text { Yield } \\
\left({ }^{\circ} \mathrm{C}\right) \\
\end{array}$ \\
\hline 1 & 11 & 20 & 41 \\
\hline 2 & 12 & 8 & 26 \\
\hline 3 & 13 & 8 & 23 \\
\hline
\end{tabular}

Isolated yield

\section{ACKNOWLEDGEMENTS}

This work was supported by the grant from Dong-A University (2012).

\section{REFERENCES}

1. T.L. Patton, J. Org. Chem., 32, 383 (1967).

2. K. Ogawa, I. Yamawaki, Y.I. Matsusita, N. Nomura, P.F. Kador and J.H. Kinoshita, Eur. J. Med. Chem.-Chim. Ther, 28, 769 (1993).

3. M. Matsui, K. Shibata, H. Muramatsu and H. Nakazumi, J. Org. Chem., 56, 4987 (1991).

4. C. Vialas, C. Claparols, G. Pratviel and B. Meunier, J. Am. Chem. Soc., 122, 2157 (2000)

5. T.J. Yu, R.G. Sutherland and R.E. Verrall, Can. J. Chem. Soc., 58, 1909 (1980).

6. L.R. Cerecedo, J. Biol. Chem., 93, 283 (1935).

7. S.K. Davidsen, G.W. Philips and S.F. Martin, Org. Synth., 65, 119 (1987).

8. G. Caraculacu, E. Scortanu and A.A. Caraculacu, J. Polym. Sci. Polym. Lett. Ed., 21, 277 (1983).

9. E. Scortanu, G. Caraculacu and A.A. Caraculacu, Iran. Polym. J., 7, 243 (1988).

10. G. Caraculacu, C. Gaina, V. Gaina and A.A. Caraculacu, Eur. Polym. J., 29, 1143 (1993).

11. G. Caraculacu, C. Gaina, A.A. Caraculacu and G. Stoica, Eur. Polym. J., 31, 987 (1995).

12. E. Scortanu, I. Nicolaescu, G. Caraculacu, I. Diaconu and A.A. Caraculacu, Eur. Polym. J., 34, 1265 (1998).

13. E. Scortanu, G. Caraculacu and A.A. Caraculacu, Rev. Roum. Chim., 43, 875 (1998).

14. J.R. Lewis, Nat. Prod. Rep., 9, 81 (1992).

15. D.J. Faulkner, Nat. Prod. Rep., 9, 323 (1992).

16. D.I. Jung, Y.Y. Kim, B.G. Yoo, Y.G. Lee and S.K. Choi, J. Korean Chem. Soc., 43, 491 (1999). 